\title{
Idiopathic intracranial hypertension in pediatric patients
}

\author{
Nad’a Jirásková \\ Pavel Rozsíval \\ Department of Ophthalmology, \\ University Hospital, Hradec Králové, \\ Czech Republic
}

Purpose: To evaluate retrospectively the features, treatment, and outcome of idiopathic intracranial hypertension (IIH) in children.

Methods: Nine patients, 15 years and younger, diagnosed with IIH. Inclusion criteria were papilledema, normal brain computer tomography or magnetic resonance imaging, cerebrospinal fluid pressure greater than $250 \mathrm{~mm} \mathrm{H}_{2} \mathrm{O}$, normal cerebrospinal fluid content, and a nonfocal neurologic examination except for sixth nerve palsy.

Results: Of the nine patients, eight were girls. Five girls were overweight and one boy was obese. The most common presenting symptom was headache (5 patients). Diplopia or strabismus did not occur in our group. Visual field abnormalities were present in all eyes, and severe visual loss resulting in light perception vision occurred in both eyes of one patient. Eight patients were treated medically with acetazolamide alone, and one girl needed a combination of acetazolamide and corticosteroids. This girl also required optic nerve sheath decompression surgery. Resolution of papilledema and recovery of visual function occurred in all patients.

Conclusions: Idiopathic intracranial hypertension in prepubertal children is rather uncommon. Prompt diagnosis and management are important to prevent permanent visual loss.

Keywords: idiopathic intracranial hypertension, pediatric, treatment

\section{Introduction}

Idiopathic intracranial hypertension (IIH), also referred to as pseudotumor cerebri, is characterized by elevated intracranial pressure without discernable etiology, with normal cerebrospinal fluid content, and normal contrast-enhanced computerised tomography (CT) or magnetic resonance irradiation (Corbett 1983; Jirásková 2000; Friedman and Jacobs 2002). Magnetic resonance imaging (MRI) can be used to predict the presence of elevated intracranial pressure. A constellation of brain MRI signs (flattening of the posterior sclera, empty sella, distension of the perioptic subarachnoid space, enhancement of the prelaminar optic nerve, vertical tortuosity of the orbital optic nerve, and protrusion of the prelaminar optic nerve) can assist in establishing the diagnosis (Brodsky and Vaphiades 1998). Idiopathic intracranial hypertension has been well described in adults, where there is a strong female preponderance and an association with obesity (Wall 1991; Liu et al 1998).

Although idiopathic intracranial hypertension occurs more frequently in adults, this condition has been well described also in the pediatric age group (Couch et al 1985). The characteristics of this disease in children may differ. In prepubertal patients, an equal sex distribution was usually found (Weisberg and Chutorian 1977; Babikian et al 1994). Affected adolescents tend to be overweight, but obesity and weight gain do not appear to be risk factors in patients under 10 years of age (Grant 1971; Baker et al 1989). Patients in whom the intracranial hypertension is not truly idiopathic but has an identifiable etiology (otitis media, dural sinus thrombosis, systemic lupus erythematosus, neck injury, metastatic disease, nephrotic syndrome or arteriovenous malformations) should be excluded. 
In this paper, we examine the clinical features of $\mathrm{IIH}$ in nine children, 15 years old and younger, with emphasis on presentation, treatment, and prognosis.

\section{Subjects and methods}

Inclusion criteria for this retrospective study were age 15 years or younger, normal brain MR imaging with or without signs of elevated intracranial pressure, intracranial pressure greater than $250 \mathrm{~mm} \mathrm{H}_{2} \mathrm{O}$ with normal cerebrospinal fluid content, edema of the optic disc, and a nonfocal neurologic examination. Patients with concurrent illnesses such as dural sinus thrombosis, otitis media, traumatic intraparenchymal or subarachnoid hemorrhage, systemic lupus erythematosus, Lyme disease and renal failure were excluded. MRI, thorough neurologic examination, routine blood tests including complete blood count, urea, electrolytes, and calcium were performed to rule out some of the associated diseases. Specific tests, for example thyroid function test, early morning cortisol, clotting studies, antiphospholipid antibodies, and Lyme serology, were done if an underlying disorder was suspected. None of the children were on vitamin A supplements, so the vitamin A level was not checked. Patients were classified into three groups: obese, overweight or normal body weight. Children were defined as obese if body mass index (calculated by dividing the weight in kilograms by the square of the height in meters) was more than 30 or body weight percentile was above the 97 th percentile in the respective age and sex population. Overweight was defined as a body mass index of 26 to 29 or weight percentile between 90 and 97 .

We reviewed the charts of all patients with the diagnosis of IIH and specifically evaluated age and symptoms at presentation, best corrected visual acuity, pupillary response, extraocular movements and diplopia, fundus biomicroscopy and optic disc findings at diagnosis and throughout follow up. Best corrected visual acuity was measured using Snellen optotypes and visual field was tested using Goldmann kinetic perimetry (Haag-Streit AG Ophthalmological Instruments, Bern, Switzerland) or Humphrey automated static perimetry (Humphrey Instruments, San Leandro, California).

\section{Results}

Nine patients ( 8 girls and 1 boy) met the inclusion criteria. Table 1 displays patient data. Mean age was $11.67 \pm 3.06$ (SD) years (range 5-15). Five girls (patients 1, 3, 4, 5, 9) were overweight and one boy (patient 7) was obese. Of these nine patients, six were symptomatic and three (patients $1,7,8$ ) were asyptomatic. In these children, IIH was diagnosed by finding papilledema on routine ophthalmic examination (one has simple myopia, one hypermetropia and one had visited ophthalmologist together with her parents). Five patients complained of headache and three of visual obscuration, one had vomiting and stiff neck. No patient had strabismus or diplopia.

A stereoscopic fundus examination was performed on all eyes. Optic disc swelling was noted in all eyes. In eight children, fully developed symmetric bilateral papilledema (engorged and dusky veins, peripapillary hemorrhages, gross elevation of the optic nerve head, choroidal folds and retinal striae) was seen. One girl (patient 9) developed asymmetric papilledema, more severe in the right eye.

The pupil and pupillary responses were assessed. Isocoria was present in all patients. The relative afferent pupillary

Table I Symptoms, examination findings, treatment, and outcome

\begin{tabular}{|c|c|c|c|c|c|c|c|c|c|c|c|c|}
\hline \multirow{2}{*}{$\begin{array}{l}\text { Pat. } \\
\text { No. }\end{array}$} & \multirow[t]{2}{*}{ Sex } & \multirow[t]{2}{*}{ Age } & \multirow{2}{*}{$\begin{array}{l}\text { Associated } \\
\text { factors }\end{array}$} & \multirow[t]{2}{*}{ Symptoms } & \multicolumn{2}{|c|}{ BCVA } & \multicolumn{2}{|c|}{ Visual field } & \multicolumn{3}{|l|}{ Treatment } & \multirow{2}{*}{$\begin{array}{l}\text { Visual function } \\
\text { recovery }\end{array}$} \\
\hline & & & & & RE & LE & RE & LE & Acetazolamide & Corticosteroids & ONSD & \\
\hline 1 & $\mathrm{~F}$ & 10 & Ovw & - & 1.0 & 1.0 & EBS & EBS & + & - & - & + \\
\hline 2 & $\mathrm{~F}$ & 12 & - & $\mathrm{HA}, \mathrm{V}, \mathrm{SN}$ & LP & LP & IT & IT & + & + & + & + \\
\hline 3 & $\mathrm{~F}$ & 15 & Ovw & $\mathrm{HA}$ & 1.0 & 1.0 & EBS & EBS & + & - & - & + \\
\hline 4 & $\mathrm{~F}$ & 12 & Ovw & TVO & 1.0 & 1.0 & EBS & EBS & + & - & - & + \\
\hline 5 & $\mathrm{~F}$ & 15 & Orw & $\mathrm{HA}$ & 1.0 & 1.0 & EBS & EBS & + & - & - & + \\
\hline 6 & $\mathrm{~F}$ & 10 & - & HA, TVO & 1.0 & 1.0 & EBS & EBS & + & - & - & + \\
\hline 7 & M & II & Obs & - & 1.0 & 1.0 & EBS & EBS & + & - & - & + \\
\hline 8 & $\mathrm{~F}$ & 5 & - & - & 1.0 & 1.0 & EBS & EBS & + & - & - & + \\
\hline 9 & $\mathrm{~F}$ & 15 & Ovw & HA, TVO & 0.5 & 1.0 & $\begin{array}{l}\text { EBS, } \\
\text { PAS }\end{array}$ & EBS & + & - & - & + \\
\hline
\end{tabular}

Abbreviations: EBS, enlarged blind spot; V, vomiting; IT, impossible to test; HA, headache; PAS, pericentral and arcuate scotomas; TVO, transient visual obscuration; Obs, obesity; ONSD, optic nerve sheath decompression; Ovw, overweight; BCVA, best corrected visual acuity; SN, stiff neck; LP, light perception. 
defect (grade one) occured only in patient 9 with asymmetric papilledema, associated with more severe visual dysfunction in the right eye. In patient 2 , the pupillary light reflex was reduced symmetrically in both eyes at presentation; in all other children pupillary reactivity was normal.

Magnetic resonance imaging demonstrated a normal brain without intraparenchymal or subarachnoid hemorrhage. In two patients, signs of elevated intracranial pressure (dilatation of the optic nerve sheaths intraorbitaly) were present. Lumbar puncture with measurement of opening intracranial pressure was performed in five children (patients $2,3,5,8,9)$. Parents of the other patients refused to give permission for lumbar puncture. Opening pressure ranged from 250 to $550 \mathrm{~mm} \mathrm{H}_{2} \mathrm{O}$ with a normal cerebrospinal fluid (CFS) composition in all patients where lumbar puncture was performed.

Visual functions were tested in all the children (Table 1). Goldmannnn or Humphrey visual fields were obtained in eight patients at presentation. In patient 2, who presented with light perception vision in both eyes, it was not possible to perform visual field testing. Visual field abnormalities were present in all eyes. Bilateral enlarged blind spot was found in seven patients. In patient 9, who presented with asymmetric disc edema, we found an enlarged blind spot and pericentral and arcuate scotomas in the eye with higher grade of papilledema, and a slightly enlarged blind spot in the eye with low-grade papiledema. Seven patients had visual acuity of 1.0 in both eyes. Two patients had decreased visual acuity. In patient 9 , visual acuity was 0.5 in the right eye and 1.0 in the left eye. Patient 2 presented with light perception in both eyes.

All patients were treated with acetazolamide (the dosing was chosen according to the weight and age of the patient) and one (patient 2) with progressive loss of vision and severe headache was treated with a combination of maximal dose of acetazolamide $(1500 \mathrm{mg} /$ day $)$ and intravenous methylprednisolone (500 mg/day for four days) followed by oral steroids. This girl also underwent an uncomplicated optic nerve sheath decompression (ONSD) in the right eye. Surgery was performed by one of the authors ( $\mathrm{NJ})$, with the patient under general anesthesia. A standard medial transconjunctival approach was used and the dura was cut by three incisions. This treatment resulted in rapid resolution of papilledema and recovery of visual functions in both eyes. The steroid therapy was gradually tapered off and the patient remained on oral acetazolamide. Six weeks after surgery, visual acuity recovered to 1.0 in both eyes, and only slightly enlarged blind spots were found on the Humphrey visual fields.
Complete resolution of papiledema and visual recovery was observed in all patients within six months. In all children, the clinical course was a single episode, with no relapse.

\section{Discussion}

Idiopathic intracranial hypertension in children and adolescents is relatively uncommon and in most cases may be associated with puberty and the resulting hormonal changes (Lessel 1992). In prepubertal children, IIH appears to be even less frequent (Cinciripini et al 1999). In our series 3 girls (patient $3,5,9)$ were in pubertal age and 6 (patient 1, 24, 6, 7 8) in prepubertal age. Children with idiopathic intracranial hypertension are thought to have an equal sex distribution (Couch et al 1985; Babikian et al 1994; Liu et al 1998; Cinciripini et al 1999). In our group, the male-to-female ratio was $1-8$.

Affected adolescents tend to be overweight, but obesity and weight gain do not appear to be risk factors in patients under 10 years of age (Youroukos et al 2000; Kesler and Fattal-Valevski 2002). In our series one boy was obese, five girls were overweight. Four of overweight girls were in pubertal age (patients $3,4,5,9$ ) and the IIH syndrome was in connection with endocrine changes. All overweight children were strongly encouraged to lose weight, but only the boy (patient 7) gradually lost $6 \mathrm{~kg}$.

Prepubertal children with idiopathic intracranial hypertension most commonly present with strabismus, which resolves rapidly with the initiation of treatment (Cinciripini et al 1999). We did not see this symptom in any of our pediatric patients with IIH. We want to emphasize that 3 children $(33 \%)$ in our group were asymptomatic and papilledema was found incidentally during eye examinations.

Visual loss has been reported to occur in children with idiopathic intracranial hypertension (Baker et al 1985; Lessell and Rosman 1986). Visual field defects were present in all eyes in this series. The most common defect in patients with idiopathic intracranial hypertension is an enlarged blind spot, which has been reported to occur in virtually all eyes with papilledema (Corbett 1983). We have found this visual field defect in almost all eyes (Table 1). Accurate visual field testing in children is sometimes difficult to perform, and hence difficult to relay on as an only accurate test. We suggest performing a kinetic perimetry in young and uncooperative children. Visual acuity less than 1.0 occurred in three eyes. Pediatric idiopathic intracranial hypertension is just as threatening to vision as the adult form (Lessell and Rosman 1986). In our study, the favorable outcome (complete vision recovery) was observed in all patients. 
Symmetric fully developed papilledema was discovered in all but one child. In patient 9 , asymmetric papilledema was found. Visual dysfunction in this girl was more pronounced in the eye with the higher grade of the papilledema as found by other authors (Wall and White 1998).

In this series, medical therapy was successful in 8 patients. In one patient with severe visual loss despite maximum medical management, an uncomplicated ONSD surgery was performed with excellent response. The safety and efficacy of this procedure in adults with visual loss due to idiopathic intracranial hypertension have been documented (Banta and Farris 2000; Jirásková and Rozsíval 2005). The experience with ONSD in children is limited, but retrospective data suggest that the safety and efficacy of the decompression surgery may be comparable with that in adult patients (Lee et al 1998; Thuente and Buckley 2005).

In summary, idiopathic intracranial hypertension in prepubertal children is less frequent than in adults or adolescents. Although pediatric patients usually present with diplopia and strabismus (Cinciripini et al 1999), we did not find this symptom in our group. Treatment of children with no or mild to moderate visual loss is primarily medical, with acetazolamide as the first-line agent. Optic nerve decompression surgery or lumboperitoneal shunt are reserved for patients with severe visual loss or progression in visual deficits under medical management. Despite rather limited experience with optic nerve sheath decompression in pediatric patients, we suggest that this procedure can be performed safely with very good outcome by the experienced surgeon, but of course more study is needed in this area. Prompt diagnosis and management are important, because children can sustain loss of visual field and acuity.

\section{Acknowledgments}

Supported in part by by research project MZO 00179906 from the Ministry of Health, Prague, Czech Republic. The authors report no financial or proprietary interest in any product mentioned.

\section{References}

Babikian P, Corbett JJ, Bell W. 1994. Idiopathic intracranial hypertension in children: The Iowa experience. J Child Neurol, 9:144-9.

Banta JT, Farris BK. 2000. Idiopathic intracranial hypertension and Optic Nerve Sheath Decompression. Ophthalmology, 107:1907-12.

Baker RS, Carter D, Hendrick EB et al. 1985. Visual loss in idiopathic intracranial hypertension of childhood: a follow up study. Arch Ophthalmol, 103:1681-6.

Baker RS, Baumann RJ, Buncic JR. 1989. Idiopathic intracranial hypertension (idiopathic intracranial hypertension) in pediatric patients. Pediatr Neurol, 5:5-11.

Brodsky MC, Vaphiades M. 1998. Magnetic resonance imaging in idiopathic intracranial hypertension. Ophthalmology, 105:1686-93.

Cinciripini GS, Donahue S, Borchert MS. 1999. Idiopathic intracranial hypertension in prepubertal pediatric patients: characteristics, treatment, and outcome. Am J Opthalmol, 127:178-82.

Corbett JJ. 1983. Problems in the diagnosis and treatment of idiopathic intracranial hypertension. Can J Neurol Sci, 10:221-9.

Couch R, Camfield PR, Tibbles JAR. 1985. The changing picture of idiopathic intracranial hypertension in children. Can J Neurol Sci, $12: 48-50$.

Friedman DI, Jacobs DM. 2002. Diagnostic criteria for idiopathic intracranial hypertension. Neurology, 95:1492-5.

Grant DN. 1971. Benign intracranial hypertension: a review of 79 cases in infancy and childhood. Arch Dis Child, 46:651-5.

Jirásková N. 2000. [Idiopatická intrakraniální hypertenze (pseudotumor mozku).] Čs Oftal, 56:262-5.

Jirásková N, Rozsíval P. 2005. [Výsledky dekompresí obalů zrakového nervu u pacientů s idiopatickou intrakraniální hypertenzí.] Cs Neurol Neurochir, 68:331-7.

Kesler A, Fattal-Valevski A. 2002. Idiopathic Intracranial hypertension in the pediatric population. J Child Neurol, 17:745-8.

Lee AG, Patrinely JR, Edmond JC. 1998. Optic nerve sheath decompression in pediatric idiopathic intracranial hypertension. Ophthalmic Surg Lasers, 29:574-617.

Lessell S, Rosman NP. 1986. Permanent visual loss in childhood idiopathic intracranial hypertension. Arch Neurol, 43:801-4.

Lessell S. 1992. Pediatric pseudotumor cerebri (idiopathic intracranial hypertension). Surv Ophthalmol, 37:155-66

Liu GT, Volpe NJ, Galetta SL. 1998. Idiopathic intracranial hypertension and its medical treatment. Drugs Today, 34:563-74.

Thuente DD, Buckley EG. 2005. Pediatric optic nerve sheath decompression. Ophthalmology, 112:724-7.

Wall M. 1991. Idiopathic intracranial hypertension. Neurol Clin, 9:73-95.

Wall M, White WN. 1998. Asymmetric papilledema in idiopathic intracranial hypertension: prospective interocular comparison of sensory visual function. Invest Ophthalmol Vis Sci, 39:134-2.

Weisberg A, Chutorian AM. 1977. Idiopathic intracranial hypertension of childhood. Am J Dis Child, 131:1243-8.

Youroukos S, Psychou F, Fryssiras S, et al. 2000. Idiopathic intracranial hypertension in children. J Child Neurol, 15:453-7. 